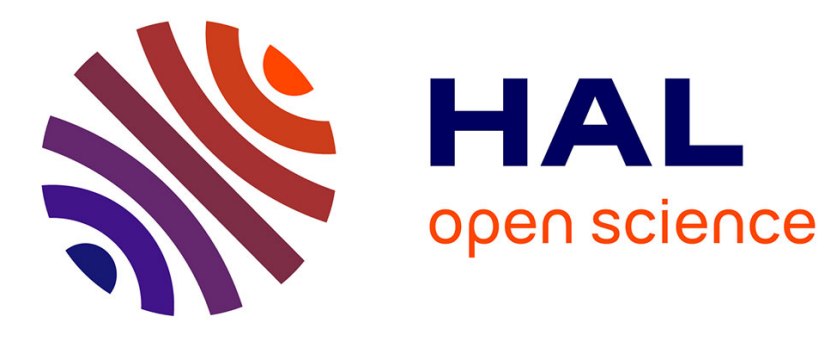

\title{
Les cadres de santé face à la logique managériale Sophie Divay, Charles Gadea
}

\section{To cite this version:}

Sophie Divay, Charles Gadea. Les cadres de santé face à la logique managériale. XIVème Journée d'études du GDR CADRES, "Encadrer sous contrainte: les encadrants des organisations publiques" IMUS, institut du management de l'Université de Savoie, Annecy décembre 2007, 2007, France. halshs-00269036

\section{HAL Id: halshs-00269036 \\ https://shs.hal.science/halshs-00269036}

Submitted on 1 Apr 2008

HAL is a multi-disciplinary open access archive for the deposit and dissemination of scientific research documents, whether they are published or not. The documents may come from teaching and research institutions in France or abroad, or from public or private research centers.
L'archive ouverte pluridisciplinaire HAL, est destinée au dépôt et à la diffusion de documents scientifiques de niveau recherche, publiés ou non, émanant des établissements d'enseignement et de recherche français ou étrangers, des laboratoires publics ou privés. 


\author{
Divay Sophie \\ Centre d'Economie de la Sorbonne
}

Gadea Charles

Laboratoire Printemps

\author{
Proposition de communication à la XIVème Journée d'études du GDR CADRES \\ « Encadrer sous contrainte : les encadrants des organisations publiques » \\ IMUS, institut du management de l'Université de Savoie, \\ Annecy, 7 décembre 2007 \\ Axe de questionnement 3 : \\ L'évolution des caractéristiques sociales des encadrants publics
}

\title{
Les cadres de santé face à la logique managériale
}

Nous allons présenter ici quelques observations issues d'une recherche en cours dans plusieurs établissements de santé (voir encadré méthodologique). Bien qu'il s'agisse d'éléments provisoires et d'une réflexion inachevée, il est clairement visible sur le terrain que la montée de la logique gestionnaire dans les hôpitaux va de pair avec une évolution de la place des cadres au sein des collectifs de travail. Ils ne sont pas les promoteurs de cette évolution, qu'ils subissent autant que les autres professionnels et que nombre d'entre eux déplorent, mais ils en sont certainement les «porteurs » au sein des équipes de travail, et les garants de sa mise en œuvre aux yeux de la direction, ce qui tend aussi à les porter sur le devant de la scène, à les charger d'une mission et les doter d'une visibilité qui n'atteignaient pas auparavant une telle ampleur.

Relativement fondus dans la masse des personnels de santé et effacés par l'omniprésence des médecins, formant traditionnellement les «patrons » de l'hôpital, les cadres de santé sont ainsi conduits à sortir de l'ombre. Ce faisant, ils révèlent leur hétérogénéité et le peu de cohésion du groupe qu'ils forment. Il semble qu'on assiste aux balbutiements d'une identité collective en émergence, selon un processus analogue à la logique historique qui avait présidé à la naissance de la catégorie des cadres dans la société française: des professions relativement disparates commencent à se fédérer sous une même bannière et à affirmer l'existence d'un être collectif parlant en son nom propre. Mais le processus est loin d'être achevé et les positions des cadres restent largement dépendantes des configurations locales, y compris à propos de ce processus de rationalisation qui les met aux premières loges de la transformation du travail hospitalier. Pendant que certains investissent avec plus ou moins d'hésitation les nouveaux espaces qui leur sont ouverts, d'autres rechignent à assumer ce rôle, en particulier parmi les cadres de soins, qui restent proches d'une défense des valeurs du service public et du métier d'infirmière, en particulier dans de petits établissements comme les hôpitaux locaux, dans lesquels on peut voir sinon des îlots de résistance à la logique 
managériale, du moins des lieux où la pression est atténuée et où les cadres contribuent à en amortir les effets sur les équipes de soin.

\section{1- Les cadres porteurs de la rationalisation?}

On peut considérer que les hôpitaux, comme d'autres services publics, sont entrés dans une phase spécifique de rationalisation qui, pour le dire en termes wébériens, ne touche pas seulement à la manière dont les moyens sont mis au service d'une fin, mais aussi à la définition des finalités mêmes de l'activité. Il existe plusieurs manières possibles de définir cette phase, souvent rapprochée du New public management ${ }^{1}$. En résumant, on passe d'un régime qui affichait pour finalité la recherche de la meilleure qualité possible des soins en tant qu'accomplissement d'une mission de service public et qui reposait sur une organisation du travail faisant une large part à la logique hiérarchisée des professions, avec, au sommet, la profession médicale, vers une situation où il s'agit d'accomplir au meilleur coût un grand nombre de tâches ou «actes », faisant l'objet d'une tarification de plus en plus précise, dans une organisation du travail où l'autonomie professionnelle ne disparaît pas, mais doit se soumettre à la prescription des objectifs et à l'allocation des moyens par des instances gestionnaires et non professionnelles. Le défi «managérial» face à la logique du professionnalisme (Freidson, 2001) est partout présent, ou du moins ressenti comme tel par les acteurs.

Dans la même période, la proportion de cadres au sein des effectifs de la fonction publique hospitalière s'accroit, en partie en raison de la requalification d'un certain nombre d'emplois de catégorie B en emplois de catégorie $\mathrm{A}$. La fonction publique hospitalière est celle des trois fonctions publiques qui compte la plus faible proportion de cadres (même en comptant les médecins), la croissance récente tient donc pour une part du rattrapage d'un retard d'évolution de la structure des qualifications. D'autre part, la fonction publique hospitalière se singularise par une catégorie B relativement plus importante que dans la fonction publique d'Etat ou la fonction publique territoriale, reflet du poids des professions intermédiaires de la santé, et notamment des infirmières, dans les effectifs. La proportion des cadres varie aussi, selon le statut des établissements de santé : les établissements publics comptent 3,1\% de cadres de santé et $1,5 \%$ de cadres de direction parmi leurs actifs, pour respectivement $1,8 \%$ et $1,8 \%$ dans les établissements privés non lucratifs et $1,4 \%$ et $1 \%$ dans le secteur privé lucratif (DREES, 2003).

A côté de cette évolution numérique, il est important de noter un changement révélateur de langage : le terme de «cadre »s'y répand de plus en plus, y compris dans le corps principal des professions du soin, où il était question auparavant de «surveillantes », «infirmières générales » ou «infirmières-chefs ». Au-delà du vocabulaire, c'est certainement la forme de catégorisation qui se transforme, selon un processus analogue à celui qui a été analysé par $\mathrm{H}$. Chéronnet (2007) dans le secteur social: les «éducateurs-chefs» et les directeurs d'établissement se voient de plus en plus souvent appliquer le terme de «cadres de l'éducation spécialisée », au moment où la rationalité managériale s'impose aussi dans ce

\footnotetext{
${ }^{1}$ Issu de modèles américains, le New Public Management calque la gestion de l'administration sur celle des l'entreprise privée. Il s'agit de définir les centres de coût, de déterminer des objectifs précis, de rationaliser les organigrammes et les cahiers des charges et de responsabiliser les centres de décision en leur donnant plus d'autonomie. Ces modèles, basés sur la notion de profit, ne font pas de distinction réelle entre service public et secteur privé. De ce fait, les citoyens sont considérés comme des consommateurs de prestations.
} 
secteur et bouscule les identités professionnelles du travail social. Plus que l'accroissement quantitatif, c'est le fait que la notion de cadre se diffuse là où elle n'avait pas cours, dans le sillage de la rationalisation managériale, qui semble fondamental et qui illustre le lien qui unit l'émergence de la catégorie des cadres et le changement de ses figures emblématiques à des phases différentes du processus de rationalisation (Gadea, 2003) : le siècle des Lumières voit naître l'ingénieur moderne (Picon, 1998), le taylorisme met en avant l'ingénieur civil de l'industrie, la modernisation d'après guerre, dans la foulée du plan Marshall et des cercles de productivité, soutient l'essor des cadres dans la société française (Boltanski, 1982). Dans la période actuelle, " la maîtrise des dépenses est devenue le principal objectif poursuivi par les autorités publiques et le débat sur la santé tend à être réduit aux enjeux économiques de la branche assurance maladie de la Sécurité sociale» (Serré, 2002, p.68), la rationalité managériale se répand dans les mondes sociaux des associations, de l'humanitaire, des arts, du travail social, là où la recherche du profit et ou même des performances économiques n'était pas un critère pertinent. Elle entraîne avec elle une redéfinition des formes de savoir et d'expertise légitimes (Bénamouzig, 2006), mais aussi un réaménagement de la division du travail et de la place des divers groupes professionnels. De même que les économistes s'imposent, au détriment des médecins, en matière de gouvernance de la santé, c'est-à-dire d'expertise et de définition des politiques publiques de santé, les cadres de santé s'imposent en matière de surveillance ${ }^{2}$, c'est-à-dire d'encadrement de proximité des soignants et de mise en œuvre des projets d'établissement, au détriment des figures de l'autorité issues des métiers emblématiques comme infirmière-chef ou surveillante ${ }^{3}$. La bataille est avant tout symbolique et se déroule souvent à l'intérieur du même individu, le poussant à agir en «homme - ou femme - de l'organisation » et non en «homme - ou femme - de la profession », et à adhérer lui-même à la logique managériale dont il est le bras séculier.

Répartition par catégorie hiérarchique des effectifs des trois fonctions publiques en 1994 et 2005 , en \%

\begin{tabular}{|l|r|r|r|r|r|r|}
\hline & \multicolumn{2}{|l|}{ Catégorie A } & \multicolumn{2}{l|}{ Catégorie B } & \multicolumn{2}{l|}{ Catégorie C } \\
\hline & 1994 & 2005 & 1994 & 2005 & 1994 & 2005 \\
\hline Fonction publique d'Etat & 37,2 & 46,6 & 32,8 & 22,5 & 30,0 & 30.9 \\
\hline Fonction publique territoriale & 7.8 & 8.4 & 12.3 & 13.7 & 79.8 & 77.9 \\
\hline Fonction publique hospitalière & 14.6 & 16.8 & 36.1 & 34.9 & 43.9 & 48.4 \\
\hline Ensemble des trois & 24.8 & 28.8 & 27.8 & 22.8 & 47.8 & 49.1 \\
\hline
\end{tabular}

Source : Rapport annuel sur l'état de la fonction publique. Faits et chiffres 2006-2007.

\footnotetext{
${ }^{2}$ Certains observateurs notent d'ailleurs une tendance au renforcement de la prédominance de la gouvernance sur la surveillance, dans le sillage des réformes contemporaines « Il semble notamment que les réformes en cours, portant une attention particulière aux objectifs gestionnaires des établissements de santé, participent à la valorisation des fonctions de gouvernance et à la relégation de la surveillance. Cette redéfinition s'opère non seulement au travers de la concentration de ces fonctions autour de postes dédiés (avec notamment les futurs chefs de pôle), mais aussi par la réorientation de certaines positions selon ces problématiques (évolution des missions confiées aux cadres de santé). La prescription et la délégation participent de ces innovations organisationnelles, puisqu'il semble qu'un objectif supplémentaire des politiques publiques soit à l'hybridation de ces relations de pouvoir avec les principes de la gouvernance : responsabilité de la gestion des pôles laissée à des médecins, contrôle accru de la gestion - par service - des ressources matérielles, etc. » N. Jounin, L. Wolf, 2006, p. 5.

${ }^{3}$ Nos observations convergent clairement avec celles d'autres chercheurs : «Ces évolutions se sont traduites dans le service public hospitalier par une recomposition importante du travail d'encadrement du travail et des témoignages suggèrent, comme on le verra, que les cadres s'éloignent de leurs fonctions soignantes, et endossent de plus en plus souvent des responsabilités de type administratif et économique, relayant les contraintes budgétaires des établissements. » Jounin et Wolf, 2006, p. 9, voir aussi Sainsaulieu 2003.
} 
Mais les effets sont bien concrets. Au quotidien, le travail et le rôle des encadrants s'en trouve modifié également. Ils sont désormais chargés de mettre en œuvre de nouvelles procédures et démarches, souvent fondées sur une approche transversale, c'est-à-dire sur le décloisonnement des services ou des unités. «Cadres de proximité » ou «cadres supérieurs », ils sont appelés à mobiliser différentes catégories de personnels afin de les inciter à prendre part à l'élaboration de projets ponctuels ou à s'investir au sein d'instances permanentes ou de groupes de travail temporaires. Ils leur revient ainsi de non seulement fédérer des salariés, mais également de leur transmettre les principes et une vision du monde hospitalier qui soustendent la rationalisation de l'hôpital public.

L'équipe de direction d'un hôpital ne saurait, en effet, conduire de nouveaux projets sans le relais actif de ses cadres. Mais ces derniers seront d'autant plus efficaces qu'ils constitueront un groupe homogène doté de connaissances communes et de représentations partagées. Or, il ne semble pas que les cadres hospitaliers forment aujourd'hui une catégorie possédant une telle unité. Ils se présentent plutôt comme un conglomérat de professions, qui peut se diviser approximativement en trois sous-ensembles: le plus conséquent est celui des cadres soignants, le plus souvent issus par promotion du métier d'infirmière ou sage-femme, le deuxième groupe, celui des cadres administratifs est plus proche de la figure classique des cadres : il comporte des comptables, des responsables de ressources humaines, des responsables de services administratifs, dans un univers où la bureaucratie reste forte; le dernier groupe est celui des cadres de la filière logistique et technique ${ }^{4}$ : la technicité croissante des soins et les flux de produits (médicaments et instruments médicaux, lingerie, alimentation, énergie, transports) ou d'informations qui circulent dans l'hôpital exigent des ingénieurs, des informaticiens, des spécialistes de la logistique, de la sécurité, etc. Par ailleurs, une certaine diversité dans les situations professionnelles est également manifeste à l'intérieur d'une même catégorie. Ainsi, les cadres soignants, hormis les différences dues à leur grade, ont des attributions et responsabilités variables selon le type d'établissement qui les emploie, voire selon les services ou les équipes qu'ils ont à encadrer.

\footnotetext{
${ }^{4}$ Au sujet de ce groupe et de sa place ambiguë dans l'hôpital, voir Metzger et Schweyr, 2005.
} 


\section{Encart méthodologique}

Cet article s'appuie sur une recherche en cours depuis 2005, lancée à la suite d'une réponse à un appel d'offres de l'ANR dans le cadre du programme « Santé-environnement et santé-travail ».

Le travail de terrain se déroule dans une même région, au sein de trois établissements de soins :

- le CHU de R. (1242 lits, personnel : 7742 équivalents temps plein [ETP] )

- la clinique M. (221 lits, personnel : 260 ETP)

- L'Hôpital local du Littoral ${ }^{5}$ (107 lits, 105 ETP)

Les monographies de ces établissements sont réalisées par observations non participante et participante, et par entretiens semi-directifs avec l'ensemble des personnels soignants, des encadrants et de la direction.

Par ailleurs, nous suivons les réunions du groupe de travail «Gestion des ressources humaines » mis en place depuis 2006 par l'ARH de la région où se situent les trois établissements de soins retenus pour le travail de terrain. Des entretiens ont également été menés avec le directeur de l'ARH, la chargée de mission qui anime le groupe GRH, ainsi qu'avec les participants (cadres soignants et administratifs, directeurs d'établissement) à ce groupe.

Pour comprendre comment s'est constituée la situation actuelle, il faut situer les transformations du présent à l'intérieur d'une histoire plus longue de l'institution hospitalière. Il n'est toutefois pas question de remonter jusqu'aux origines du «grand renfermement» (Foucault, 1961) et aux différents styles de gestion dont les hôpitaux ont fait l'objet au cours de leur histoire, nous esquisserons simplement ici un rapide tableau des grands tournants qui ont mené à l'instauration de la logique managériale.

\section{1- 1- Rationalisation progressive du fonctionnement de l'hôpital}

Réservé aux pauvres, c'est-à-dire dédié à l'accueil des indigents et vagabonds, jusqu'au début du XXe siècle, l'hôpital ne s'ouvre que progressivement à l'ensemble de la population. Son instauration en tant qu'établissement de soins se poursuit avec la création du corps des directeurs d'hôpital en 1941 et à travers la mise en place d'une spécialisation progressive des services d'hospitalisation en fonction de classes particulières de pathologies. La période de croissance économique permet aux hôpitaux de se développer à un rythme soutenu qui se traduit par une multiplication par quatre des dépenses totales en 1960 et 1970, exprimées en francs constants (Moisdon, Tonneau, 1999).

Un tournant s'amorce avec la planification sanitaire et l'encadrement des dépenses introduits par la loi hospitalière du 31 décembre 1970 qui crée la notion de "Service public hospitalier" et instaure la carte sanitaire, découpage de l'espace géographique visant une meilleure répartition des équipements hospitaliers. Jusqu'à la fin des années 1970, les établissements publics demeurent sous le principe de l'approbation par l'autorité de tutelle des décisions de leur conseil d'administration.

La maîtrise des dépenses de santé et la transformation des modes de régulation du secteur hospitalier interviennent à partir des années 1980, à la suite de l'arrêt de la croissance et des crises répétées du système de protection sociale qui conduisent l'Etat à imposer à l'hôpital une régulation par contingentement des ressources. En 1984, est instauré le système de dotation globale par lequel le budget des établissements est déterminé a priori. Cette

\footnotetext{
${ }^{5}$ Cet établissement porte ici un nom d'emprunt.
} 
délégation des responsabilités économiques par la mise en place d'un budget global contraint les directeurs d'établissement à acquérir une plus grande qualification gestionnaire. Ces derniers doivent désormais maîtriser des systèmes d'information médicalisés et réaliser des économies sur l'ensemble des postes de dépenses.

L'orientation gestionnaire se renforce depuis le début des années 1990. La tutelle hospitalière tente en effet de mettre en place des dispositifs de régulation de plus en plus fins, la procédure de la dotation globale ayant montré ses limites, notamment au niveau de l'inéquité potentielle de l'allocation des ressources et de la possibilité de rentes de situation. Un remède à ces dérives est appliqué à travers une redistribution des ressources fondée sur de nouveaux outils de formalisation de l'activité hospitalière désormais gérée de façon plus qualitative par l'élaboration de projets internes aux établissements. La loi de réforme hospitalière de 1991 encourage ainsi la participation de l'ensemble des personnels à l'élaboration du projet d'établissement dans le cadre de Conseils de services et renforce la représentation des personnels. Cette loi instaure par ailleurs le Schéma régional d'organisation sanitaire (SROS) qui prend désormais en compte la dimension régionale en tant que référence sanitaire. Le pouvoir de l'Etat est pour partie délégué aux instances régionales ou locales. En matière de planification, la réforme issue de l'ordonnance du 24 avril 1996 institue les Agences régionales de l'hospitalisation (ARH) et régionalise les budgets avec pour objectif d'améliorer la complémentarité de l'offre de soins au sein d'une même zone géographique. L'ARH est notamment chargée de la contractualisation des établissements hospitaliers. On assiste alors à un transfert des pouvoirs de gestion budgétaire et de régulation de l'activité vers l'échelon régional et les établissements avec toutefois le maintien d'une forte centralisation en ce qui concerne l'emploi.

Dans le cadre du plan Hôpital 2007, l'ordonnance du 4 septembre 2003 simplifie et régionalise les démarches de planification : la carte sanitaire est supprimée, le SROS devient l'outil unique de planification, les "secteurs sanitaires" sont remplacés par des "territoires de santé", toutes les autorisations sont déconcentrées au niveau des ARH. Par ailleurs, l'ordonnance unifie les formules de coopération sanitaire entre établissements publics et privés en faisant du Groupement de Coopération Sanitaire (GCS) le cadre naturel des coopérations et des réseaux de santé.

Ces grandes directives reposent sur une décentralisation administrative, l'adoption de principes de marché et la contractualisation. Concrètement, les établissements de soins fonctionnent désormais sur la base d'une gestion par objectifs centrée sur l'efficience, la performance et la qualité, qui implique la mise en œuvre de nouvelles procédures et démarches de plus en plus formalisées, fondées sur des méthodes de travail transversales. Les cadres sont ainsi considérés comme des acteurs moteurs chargés d'assurer le développement des démarches qualité concernant entre autres l'hygiène, la prise en charge de la douleur ou les soins palliatifs. Ils ont également la responsabilité de mettre en application et de suivre l'actualisation des réglementations liées à la gestion des risques (hémovigilance, matériovigilance, pharmacovigilance...). Il leur faut encore animer des groupes de travail consacrés à l'élaboration du projet d'établissement ou à la conduite de la démarche d'accréditation. Ces nouvelles attributions sont intervenues comme des facteurs d'évolution et de redéfinition du rôle des cadres soignants. 


\section{1- 2- Les incidences de la rationalisation sur les cadres de santé}

Les cadres hospitaliers n'ont fait l'objet que de rares analyses et, à notre connaissance, celles qui ont été réalisées ont surtout porté sur les cadres de soins, sans doute parce qu'ils sont numériquement majoritaires parmi l'ensemble des cadres à l'hôpital et de ce fait plus visibles $^{6}$. Ce paragraphe s'appuie en grande partie sur les travaux qu'Isabelle Féroni et Anémone Kober-Smith ont mené sur les cadres infirmiers et qu'elles ont présenté dans un article de la Revue française de sociologie en 2005.

L'histoire de l'encadrement infirmier connaît une évolution certaine au tournant des années 1960. Comme cela a été souligné précédemment, cette période se caractérise par un fort développement de l'activité des hôpitaux qui se modernisent rapidement. Leur fonctionnement traditionnel est remis en question par une technicisation et une spécialisation des services. Cette complexification médico-technique entraîne une division du travail accrue entre les catégories de personnel qui génère un besoin de coordination entre les unités. Les fonctions d'encadrement évoluent et sont de plus en plus axées sur le management du personnel. Parallèlement, «l'aspect polyvalent de la fonction de surveillante et sa dimension domestique s'effacent» (Féroni, Kober-Smith, 2005). Cette catégorie de cadres parvient progressivement à gagner une plus grande autonomie professionnelle. La création en 1975, du poste d'infirmière générale, cadre $\mathrm{A}$ de la fonction publique, place désormais ces encadrantes sous l'unique autorité du directeur d'hôpital, alors que précédemment les surveillantes d'unité de soins et les surveillantes générales de pavillon dépendaient également de l'autorité du médecin-chef.

Une ligne hiérarchique est clairement établie et s'échelonne sur trois grades : surveillante d'unités de soins, surveillante-chef et infirmière générale que la loi de réforme hospitalière de 1991 désigne comme membre à part entière de l'équipe de direction hospitalière. A partir de ce moment, la profession médicale n'intervient plus dans les procédures de recrutement des surveillantes. Ces titres vont progressivement être modifiés. Vers 1985, l'appellation de « surveillante » est remplacée par celle de «cadre infirmier» pour devenir «cadre de santé » en 1995 (Acker, 2003). Par ailleurs, en 2002, le statut de «directeur des soins » supplante celui d' « infirmier général » ${ }^{7}$.

\footnotetext{
${ }^{6}$ A titre d'exemple, dans le CHU où nous menons une partie de notre travail, les effectifs des personnels se répartissent comme suit :

- total du personnel non médical : 7066 personnes dont 460 cadres toutes filières confondues,

- part de cadres soignants : $66 \%$.

7 Tableau récapitulatif de la hiérarchie paramédicale à l'hôpital public (source : Vega, 2004) :
}

\begin{tabular}{|c|c|}
\hline \multicolumn{2}{|c|}{ Hiérarchie para médicale } \\
\hline Anciens titres hiérarchiques & Titres actuels \\
\hline $\begin{array}{c}\text { Infirmière générale } \\
\text { Chargée de l'encadrement du personnel infirmier, } \\
\text { de l'organisation des plannings, de la gestion du } \\
\text { matériel et des relations avec la direction }\end{array}$ & Directeur des soins infirmiers \\
\hline $\begin{array}{c}\text { Surveillante chef ou surveillante générale } \\
\text { Cadre infirmier supérieur }\end{array}$ & Cadre de santé supérieur \\
Responsable d'un service & Cadre de santé \\
\hline $\begin{array}{c}\text { Surveillant de soins } \\
\text { Cadre infirmier } \\
\text { Responsable d'une partie d'un service ou d'une } \\
\text { unité }\end{array}$ & \\
\hline
\end{tabular}


Ces évolutions terminologiques ne sont pas neutres. Elles traduisent l'éloignement de ces cadres par rapport à leur métier d'origine et leur rapprochement des fonctions plus généralistes de gestion, notamment à travers l'abandon du terme «infirmier». Ces anciennes spécialistes du soin sont contraintes d'adhérer à une nouvelle définition de leur rôle professionnel, celui de manager au sein du Service public.

Cette tendance a été renforcée par la mise en place du Plan Hôpital 2007. Cette mesure introduit une «nouvelle gouvernance hospitalière» en créant notamment les «Pôles d'activités », nouvelles entités juridiques destinées à regrouper et restructurer les services et unités fonctionnelles déjà existants au sein d'un établissement. Selon l'ordonnance du 2 mai 2005, ces pôles sont institués par un Conseil de pôle présidé par un médecin coordonnateur assisté par des cadres de santé et des cadres administratifs. Les cadres devront encourager les personnels hospitaliers à participer au fonctionnement de ce Conseil de pôle, notamment en organisant l'élection de représentants au sein de chaque catégories de personnel.

Les Pôles d'activités disposent d'une marge de manœuvre conséquente dans le sens où le directeur général de l'établissement délègue une grande responsabilité de gestion au chef de pôle au moyen de procédures de contractualisation interne. Le contrat, négocié puis cosigné par le directeur général, le président de la Commission médicale d'établissement et le responsable de pôle, définit les objectifs d'activités, de qualité et financiers, les moyens et indicateurs de suivi des pôles, les modalités d'intéressement et les conséquences en cas d'inexécution du contrat. Sans entrer dans le détail de l'organisation des Pôles d'activités, il apparaît clairement que des fonctions de gestion comptable sont de plus en plus attribuées à des personnels médicaux et paramédicaux «de terrain». Médecins, cadres infirmiers et administratifs ne peuvent désormais pas échapper aux fonctions de management. Les propos d'une chef du personnel, rapportés par Ivan Sainsaulieu, traduisent bien cette «managérialisation» de tous les personnels de l'hôpital et la généralisation du modèle entrepreneurial : "Cela devient difficile de faire des choix. Je trouve cela moche. Durant la période des sept dernières années, la contrainte budgétaire a été très forte, on n'avait pas l'habitude, j'ai connu des budgets terribles, il a fallu qu'on rende des comptes à nos concitoyens. La mini-révolution, cela a été de se découvrir gestionnaire, petits chefs d'entreprise à part entière. On est arrivé à une situation où l'on a bien appris, avalé cette notion, les efforts ont été faits, et maintenant on ne peut plus aller plus loin. »Chef du personnel, Hôpital pour enfants (Sainsaulieu, 2003, p. 61).

Comment réagissent les cadres à cette transformation du contexte et du contenu de leur travail, mais aussi de leur position dans l'hôpital ? Nous ferons principalement appel pour apporter des éléments de réponse aux observations réalisées dans deux des sites de notre enquête de terrain, le CHU de R. et l'Hôpital Local du Littoral, dont les configurations contrastées permettent de mieux cerner la diversité des situations professionnelles des cadres hospitaliers. Dans l'un des cas, on verra se mettre en place une tentative de réponse via l'organisation collective des cadres aux interrogations que suscitent les réformes hospitalières; dans le deuxième, c'est plutôt en tentant de préserver des relations professionnelles fondées sur la proximité avec l'équipe de soins que la cadre de l'hôpital local fait face à ces réformes, en accord avec un directeur peu sensible au chant des sirènes managériales. 


\section{2- Un CHU face à l'hétérogénéité des encadrants hospitaliers : l'expérience du « Collège cadres »}

Le CHU de R. est entré avec un temps d'avance dans l'ère de la nouvelle gouvernance. Il a en effet fait partie, en 2004, des quatorze CHU (sur trente et un) retenus par le Ministère pour mettre en œuvre, par anticipation, les pôles d'activités. En tant qu'établissement pilote, il a reçu une dotation de 80000 euros pour financer l'intervention de consultants chargés d'accompagner la mise en place des nouvelles instances prévues par les textes officiels et la restructuration organisationnelle des services. Une des priorités a été d'instaurer une contractualisation interne et des négociations entre médecins sur les éventuels rapprochements possibles entre disciplines et services, dans le but de créer les Pôles d'activités ${ }^{8}$. Ces appariements viennent bouleverser une organisation établie de longue date et soulèvent des questions cruciales, telles que la répartition des moyens humains, financiers et matériels par Pôle ou encore celles des critères à appliquer : faut-il, par exemple, continuer à se référer au nombre de lits ? Il s'agit entre autres de déterminer le mode d'évaluation de l'optimisation des ressources. Les médecins se trouvent ainsi confrontés à des prises de décisions auxquelles ils n'avaient jusqu'à présent jamais été associés. Ils ne sont toutefois pas seuls dans ce cas. Les cadres de proximité et intermédiaires sont également appelés à prendre part à cette réorganisation puisque les textes prévoient que les chefs de Pôle, ou médecin coordonnateur, doivent être assistés de cadres de santé et administratifs. La direction de l'établissement a de ce fait intérêt à mobiliser les encadrants et doit compter sur eux pour relayer les principes gestionnaires de la nouvelle gouvernance auprès des personnels des différentes catégories.

Un concours de circonstances a en quelque sorte servi ces intentions. En effet, en 2002, le nouveau directeur général décide, à son arrivée, de réunir son équipe de direction élargie (directeurs de tous les départements) et de lancer une démarche projet d'une durée d'un à deux ans. La consultation de ses collaborateurs fait notamment émerger un questionnement autour du rôle de l'encadrement, qui fournira un des axes de réflexion auquel seront conviés les cadres. Les changements qui s'annonçaient à l'hôpital laissaient supposer un repositionnement du corps de direction et des cadres, suscitant ainsi de nombreuses incertitudes et un certain malaise chez les encadrants : que signifie être cadre hospitalier au début des années 2000 dans un établissement de santé ? Quelles sont les missions d'un cadre ? Certains cadres éprouvaient un déficit de reconnaissance et jugeaient leur position inconfortable, parce que confrontés à des contradictions, telles que l'obligation de gérer des équipes, sans être vraiment maîtres de la situation notamment du fait de la forte réglementation des emplois dans la fonction publique. Par ailleurs, ils ne s'estimaient pas suffisamment informés, en tout cas souvent moins que les syndicats, alors qu'on leur demandait de plus en plus d'accompagner, voire de porter, les changements en cours.

Face à ces doléances, le directeur général instaure officiellement, début 2004, un «Projet encadrement », conduit par un Comité de pilotage (Copil) composé d'une quinzaine de cadres et de cadres supérieurs des trois filières soignante, administrative, technique et logistique. Il charge en outre la directrice adjointe de la Direction de l'analyse, de la prospective et des activités médicales (DAPAM) de lui rendre compte régulièrement des travaux de ce Copil. La désignation de cette intermédiaire ne se fait pas au hasard. En effet, cette directrice adjointe travaille en lien étroit avec la tutelle, c'est-à-dire l'AHR, et à ce titre prend une part active dans la rédaction du Projet d'établissement et du Contrat pluriannuel d'objectifs et de moyens (CPOM). Ces deux outils de gestion clé, parce que sources de financements, supposent une forte mobilisation des cadres chargés de faire remonter les informations et d'impliquer les

\footnotetext{
${ }^{8}$ Les 54 services existants au sein du CHU de R. ont été regroupés en 11 Pôles d'activités.
} 
personnels dans les orientations politiques et transversales de l'établissement. Cette directrice se trouve donc «naturellement » encline à soutenir le Projet encadrement dont l'activité s'est déployée à travers la création de plusieurs groupes de travail impliquant au total plus de 160 cadres volontaires. Ces derniers remettent un rapport final au directeur général et en présentent le contenu en septembre 2005 devant plus de 300 cadres (sur un total de 460). La proposition de la création d'un «Collège cadres » retient particulièrement l'attention du directeur général qui autorise sa création et organise son fonctionnement : ce Collège reçoit un mandat de deux ans et est composé de vingt et un membres tirés au sort parmi un ensemble de cadres volontaires.

Le directeur général a donc pleinement soutenu la mise en place d'un Collège cadre qu'il a considéré comme le moyen de faire émerger un acteur collectif, interlocuteur et relais indispensable à la diffusion de la pensée managériale au sein de son établissement. Alors que la directrice adjointe de la DAPAM, nous a tout d'abord présenté l'introduction des Pôles d'activités et la création du Collège cadre comme deux aspects indépendants, elle reconnait, en fin d'entretien, qu'un lien les unit : «Le sujet de tout le Projet encadrement, c'est quand même : comment on associe davantage à la conduite du changement les cadres ? Qu'est-ce qu'on attend demain d'un cadre hospitalier ? Comment on le positionne au mieux ? D'accord, il faut qu'il soit associé à la décision, mais il faut aussi qu'il porte la décision ! Y a les deux aspects : côté cadres, on peut se dire : "Bah, effectivement, j'ai envie d'être associé plus, je le suis pas assez !" Et puis, côté direction, on se dit aussi, quand ça vous remonte parce que c'est des contextes difficiles, vous vous dites : "Attendez! Les messages, ils sont portés ou pas par l'encadrement ? Jusqu'à quel point c'est expliqué ?" Oui, ça se rejoint assez naturellement, c'est pas le fait du hasard complètement. Avec le Collège cadres, on aurait pu rester sur quelque chose d'assez formation, référentiel de métier... » (directrice adjointe du DAPAM).

Etant donné l'intérêt que les cadres ont manifesté pour le Projet encadrement, puis sa transformation en Collège cadres, le directeur général et la directrice adjointe de la DAPAM s'attendaient à ce que les membres de ce Collège trouvent spontanément un mode de coopération harmonieux; or, l'expérience montre qu'il leur faut en quelque sorte un temps d' «acclimatation »: " On se dit, ça va aller vite parce qu'ils sont tous cadres, il y a quand même les mêmes valeurs, y a un socle... Et là, vous vous rendez compte que entre un cadre administratif, un cadre soignant, c'est pas la même représentation. Le temps que chacun appréhende : "Ah bon, et toi tes contraintes, c'est ça !" Et là, on voit que ça prend du temps de s'approprier ce groupe-là. Il faut qu'il crée son identité, ça prend du temps, les gens ne se connaissaient pas et les métiers des uns et des autres n'étaient pas forcément... Vous mettez un cadre à un moment donné soignant qui se retrouve dans un Collège cadres avec l'attaché qui est à la DRH, et qui explique son métier et voilà quels sont... Eh bah, vous comprenez mieux que les contraintes des uns et des autres ne sont pas connues ! (Directrice adjointe du DAPAM)

Ces quelques lignes résument les effets produits par une expérience certes appréciée des cadres, mais qui n'est pas sans brusquer leurs habitudes. En effet, ils n'avaient, avant la mise en place du Collège cadres, jamais eu l'occasion d'être rassemblés pour participer à une même réunion. Certains travaillaient au CHU depuis des années sans s'être jamais rencontrés. Les cadres sont en quelque sorte cantonnés dans leur filière, pour ne pas dire parfois dans leur service ou département. Si la tentative de décloisonner les trois filières cadres et de faire naître une catégorie plus unifiée et homogène d'encadrants n'a pas encore atteint son but, elle a cependant donné vie à un groupe légitime et représentatif de porte-parole des cadres à travers le Collège cadres. L'entrée en jeu de ce nouvel acteur collectif, doté d'une certaine 
force de négociation du fait de sa représentativité, n'est pas sans générer certaines tensions. Les syndicats s'interrogent, par exemple, sur le poids que peut prendre ce Collège aux côtés de représentants élus, notamment lors des tractations et pourparlers avec la direction. Par ailleurs, la Direction des ressources humaines (DRH) voit ses prérogatives bousculées. Elle a en effet accepté que figure désormais au catalogue de la formation continue, une rubrique « formations tous cadres », alors qu' auparavant les stages proposés étaient destinés à tous les personnels sans distinction de catégorie. La DRH a donc été dotée d'une enveloppe spécifique pour ces formations de cadres. Les membres Collège cadres ont récemment fait part de leurs desiderata : ils souhaitent en effet pour 2007 que des formations à la gestion du stress soient organisées et, en outre, que ces stages aient lieu en dehors de l'établissement. Face à cette demande, la directrice des ressources humaines s'est sentie dépossédée de ses prérogatives, car elle n'a pu refuser de mettre en place ce projet, même s'il demande de faire appel à de nouveaux organismes de formation et s'avère plus onéreux. Par ailleurs, les membres de la direction des soins ont exprimé leur inquiétude face à ce nouveau Collège cadres dont ils mesurent mal le pouvoir d'action et de décision actuel et futur. La mise en place des Pôles d'activités suscite à l'heure actuelle beaucoup de questions. Les cadres soignants ignorent la place qu'ils pourront réellement occuper dans cette reconfiguration des services. Qui pourra prendre part aux décisions aux côtés du médecin coordonnateur? Le Collège cadres aura-t-il voix au chapitre dans cette répartition des pouvoirs? A ce propos, la Direction générale s'interroge aujourd'hui sur le positionnement des membres du Collège cadres : par exemple, comment un «cadre finances » peut-il objectivement représenter les intérêts de l'ensemble des cadres?

Le directeur général est donc confronté aux effets secondaires d'une décision stratégique censée faciliter la diffusion de la nouvelle gouvernance au sein de son établissement. A l'heure actuelle, la constitution d'une entité collective des cadres demeure balbutiante, mais les membres du Collège cadres commencent, pour leur part, à faire valoir leurs intérêts en s'emparant du pouvoir qui leur a été accordé. Ils ont sans doute compris que la Direction générale a, dans le contexte actuel, tout particulièrement besoin d'eux. Elle compte par exemple sur leur participation pour définir le profil du cadre administratif qui selon les textes doit faire partie de chaque Conseil de Pôles auprès du médecin coordonnateur. Le directeur général se trouve aujourd'hui face à un noyau d'acteurs cadres, stratégiquement utile, mais dont l'existence risque d'être contestée par certaines instances (syndicats) ou directions (DRH, Directions des soins...), susceptibles de les considérer comme des rivaux. Selon la directrice adjointe de la DAPAM, de telles réactions pourraient être contrées par l'organisation d'élections des membres du Collège cadres qui serait ainsi pourvu d'une plus grande légitimité.

A l'échelle d'un CHU, la mobilisation des cadres et leur fédération en une catégorie homogène n'est pas sans poser problème du fait, d'une part, de leurs divisions internes organisationnelles, mais aussi, d'autre part, du fait des perturbations que leur entrée dans le jeu des rapports de pouvoir peut générer. Il en va tout autrement dans un Hôpital Local qui constitue pour ainsi dire un cas de figure opposé à celui d'un CHU : l'équipe de direction est réduite à sa plus simple expression avec un directeur et une cadre de santé ; la nouvelle gouvernance ne peut s'appliquer sans certains ajustements locaux; le courant de rationalisation se heurte aux résistances d'une organisation traditionnelle fortement ancrée. 


\section{3- L’Hôpital Local : un îlot de résistance face à la logique managériale ?}

L'Hôpital Local du Littoral est implanté à SPC, petite ville côtière de 5000 habitants. Ancien hospice, il a été rénové au début des années 1980 et comporte aujourd'hui trois services de 107 lits au total. A l'étage, sont regroupés en deux unités les 67 pensionnaires de l'EHPAD'. Le rez-de-chaussée comprend un service de médecine de 18 et lits et une unité de 22 lits réservée à des personnes handicapées mentales vieillissantes en long séjour.

Un Hôpital Local a pour vocation principale d'offrir une plate-forme sanitaire et sociale de proximité (cf. Circulaire de mai $2003^{10}$ ), souvent dans une zone géographique rurale. Cet établissement ne compte pas de personnel médical parmi ses salariés et les malades sont de ce fait suivis par leur médecin généraliste qui se déplace à leur chevet. Une partie de ces médecins, installés en cabinet privé dans un rayon d'une trentaine de kilomètres de l'Hôpital, siègent à la Commission médicale d'établissement (CME). Par ailleurs, le Conseil d'administration est présidé, selon les textes réglementaires, par le maire de la commune où l'Hôpital est situé. Ces quelques aspects organisationnels ne laissent qu'entrevoir le poids du local dans le fonctionnement d'un tel établissement. En effet, tous les membres de l'Hôpital (soignés, soignants, et autres personnels du haut en bas de la hiérarchie) signalent très vite au nouveau venu, comme pour le plonger d'emblée dans l'ambiance, la caractéristique principale des lieux : «Ici, c'est familial ! Tout le monde connaît tout le monde. » Cette formule résume la force de ce milieu d'interconnaissance où personne n'est vraiment un inconnu pour les autres : les liens sont familiaux, de voisinage, ancrés dans le passé, amicaux ou conflictuels, interconnaissance ne signifiant pas harmonie (Beaud, Weber, 1997, p. 296). A titre d'exemple, un nouveau patient est, bien avant son arrivée, l'objet d'un processus d'étiquetage. En effet, les dossiers ou simplement les noms et prénoms des nouveaux entrants sont souvent transmis dans le service quelques jours avant leur admission. Dans ce cas, quelqu'un est toujours en mesure de livrer des informations sur ce patient : «Ah, oui, c'est Mme X, je la connais bien, c'est la mère d'un ami de mon mari... » L'informatrice ${ }^{11}$ dresse alors un tableau de cette personne qui permet à ses collègues de construire une image du futur patient, par avance classé dans la catégorie «bon »ou « mauvais malade».

Le fait d'être du coin (Renahy, 2005) ou, autrement dit, ce principe d'autochtonie (Retière, 2003) concerne également les deux derniers directeurs de l'établissement. Mr Legrand ${ }^{12}$, directeur actuel, âgé de 54 ans, est né à SPC et a occupé son premier emploi à l'Hôpital du Littoral. Parti du bas de l'échelle, il a progressivement gravi les échelons, en passant des concours qui l'ont amené à changer plusieurs fois d'établissement. C'est en 1994 qu'on lui propose la direction de l'Hôpital du Littoral où il compte aujourd'hui finir sa carrière. Il aurait la possibilité, s'il le souhaitait, de progresser encore en acceptant une mutation au CHU de R., mais cette perspective ne le tente pas. La hiérarchie dans tel établissement lui semble trop lourde et il préfère de ce fait rester dans l'Hôpital Local du Littoral où les relations sont, d'après lui, bien moins protocolaires. Les caractéristiques de son parcours et de l'établissement qu'il dirige déterminent en partie son style de management (terme qu'il n'emploie guère). Il tient de toute évidence à garder une distance avec le personnel pour conserver une certaine autorité et pour se protéger d'une forme de familiarité qui risquerait de s'installer spontanément. Nombre de salarié(e)s l'ont en effet connu quand il était jeune,

\footnotetext{
${ }^{9}$ EHPAD : Etablissement d'hébergement pour personnes âgées dépendantes.

${ }^{10}$ Circulaire : DHOS/DGAS/O3/AVIE/n²003/257 du 28 mai 2003, relative aux missions de l'Hôpital Local.

${ }^{11}$ Le féminin est utilisé pour désigner le personnel soignant composé uniquement de femmes.

${ }^{12}$ Pseudonyme.
} 
certains l'ont vu débuter dans l'établissement, d'autres ont fréquenté la même école que lui ou habité dans le même quartier. Toutefois, il reste en contact direct avec le quotidien de l'établissement. Il a par exemple l'habitude de passer presque tous les matins un moment dans la salle d'animation où les pensionnaires valides se réunissent. Il s'installe à une table, boit un café, discute avec les animateurs et laisse les pensionnaires venir à lui : «Bon, puis les gens dans les résidents se gênent pas, hein ! Comme ils me connaissent bien, donc, comme tous les jours je monte là haut, je suis au courant de tout ! Même parfois des potins personnels. Y a bien un résident qui me le glisse dans l'oreille... Moi, je lui dis : "C'est bien, merci !" Donc, (rire) quand ça va pas, je le sais ! Cette méthode n'est pas sans déplaire à certains agents qui relève à leurs yeux d'un contrôle informel dangereux car incontrôlable et parfois suivi de retombées pour certaines d'entre elles.

Mr Legrand reconnaît qu'il se démarque des directeurs de la «nouvelle génération», beaucoup plus gestionnaires dans l'âme que lui. Lorsqu'il se retourne sur son passé, il mesure à quel point le milieu hospitalier s'est transformé. Entre le moment de ses débuts, époque à laquelle on parlait d'hospice et où les «bonnes sœurs» étaient encore en fonction, et aujourd'hui, une véritable révolution s'est produite. Il ne regrette pas les dortoirs de quarante lits (appelés les «mouroirs ») et reconnaît les progrès réalisés en termes d'hygiène et de sécurité, mais il déplore la bureaucratisation du fonctionnement de l'hôpital ainsi que le durcissement et la démultiplication des contraintes de la tutelle. Les exigences budgétaires se sont accentuées et plus aucune négociation ne semble possible : «Il y a encore sept ou huit ans, j'allais à la DDASS, je prenais rendez-vous, on discutait du fonctionnement de l'hôpital, on discutait des orientations, on discutait du budget. Aujourd'hui, on ne discute plus ! J'y vais plus! On me dit: "C'est pas la peine !" Les décisions sont prises. C'est ce que je ressens, hein ! On discute plus : "Voilà votre enveloppe !". Ah, on a beau dire : "Mais attendez, on a des fonctionnements sur votre enveloppe..." Point ! La seule autorité avec qui je peux encore discuter, c'est le Conseil général. Parce que c'est des gens de ma génération. Combien de fois, j'ai dit: "Encore heureux que vous êtes là, parce que la génération future..." Donc, vous voyez, déjà, la nouvelle génération des jeunes inspecteurs DDASS... Voilà, voilà, là, on vous envoie votre notification budgétaire, et puis, bah, vous vous débrouillez avec ça !»

Les effets de la mise en place de l'ARH se sont faits sentir, il y environ six ou sept ans. Cette nouvelle autorité régionale a, selon Mr Legrand, « renforcé le pouvoir décisionnel ». En tant que directeur, il dit être confronté à des contradictions ou des décalages entre les discours et les faits. Si le mot d'ordre est depuis quelques années celui de la décentralisation, les attentes et injonctions de l'ARH vont dans le sens d'une plus grande centralisation, à un niveau régional. Cette instance a désormais «la totale maîtrise des dépenses » et n'autorise aucune souplesse de gestion aux établissements. La logique managériale leur est imposée et les responsables doivent faire face à une inflation de procédures qui s'accumulent au fil des ans, pour en arriver parfois à une situation ubuesque : "On est en train de gérer les hôpitaux comme les entreprises privées ! (...) Et on me dit: "Il faudrait faire une Commission des relations des usagers." OK, c'est bien! "Où vous en êtes dans votre CLAN ?" C'est quoi le CLAN ? Comité de liaison alimentation et nutrition. Ah, oui, ça c'est bien ! "Il va peut-être falloir penser à constituer un CLUD ${ }^{13}$ !". (rire)... contre la douleur... mais, mais.... c'est dingue! "Allez-vous faire un CLIN ${ }^{14}$ ?" Oui, le CLIN, oui ! J'ai dit, mais, comment je vais faire pour constituer tout ça ? Avec qui ? Le problème, c'est toujours avec qui ! Et puis après, il faut faire des réunions... parce que entre temps, bah, vous avez l'accréditation. Donc, avec des experts... qui vous disent: "Votre CLIN, ça fonctionne ? Oui ? Bah, donnez-moi une

\footnotetext{
${ }^{13}$ CLUD : Comité de lutte contre la douleur.

${ }^{14}$ CLIN : Comité de lutte contre les infections nosocomiales.
} 
pièce justificative !" Donc, on ne peut pas faire autrement que de se réunir, même si on a rien à se dire ! »

A lui seul, le directeur n'a ni le temps, ni les moyens d'assurer le fonctionnement de tous ces groupes de travail et instances. Il s'appuie sur ce qu'il appelle sa «garde rapprochée » dont le membre essentiel est l'unique cadre de l'établissement, Mme Joly, cadre infirmier, plus communément nommée la surveillante ou la «susu ». C'est à elle que le directeur délègue une grande part de responsabilités. Elle joue un rôle d'intermédiaire entre la direction et le personnel soignant, les médecins, les patients et les familles, sans compter les contacts avec les partenaires du tissu socio-médical local.

Issue d'une famille d'ouvriers, Mme Joly a aujourd'hui 54 ans. Elle est née en région parisienne, où elle a obtenu son diplôme d'infirmière et où elle a exercé sa profession dans plusieurs établissements, notamment en gériatrie et dans un service de réanimation. Elle obtient en 1985 son diplôme de cadre infirmier et enseigne pendant cinq années dans l'école d'infirmière du CHU de R., où elle prend, après cette expérience d'enseignement, un poste de cadre en pneumologie. Les relations avec sa supérieure hiérarchique s'avérant très tendues, elle décide de chercher un autre emploi et postule à l'Hôpital Local du Littoral où la direction de l'époque avait décidé de créer un poste de surveillante pour mettre fin à des conflits de pouvoir existant au sein de l'équipe soignante: «J'ai été extrêmement bien accueillie ici parce que l'équipe souffrait énormément d'un espèce de contre-pouvoir soignant. C'est-à-dire que le pouvoir ici était essentiellement administratif, il y avait jamais eu de cadres soignants ici. (...) Donc ce poste, il est ce que j'en ai fait, bien ou mal, il est ce que j'en ai fait !» (Mme Joly).

Afin de trouver sa place dans l'établissement, Mme Joly a dû tout d'abord apprendre à connaître professionnellement et personnellement l'ensemble des salariées. Bien qu'étant extérieure au réseau d'interconnaissance locale, elle est parvenue à se faire accepter à force de patience et d'écoute : «j'ai surtout beaucoup écouté, beaucoup de réunions d'équipe où j'ai écouté tout le monde... Je passais donc un soir par semaine pour discuter avec l'équipe de nuit, ce que je ne fais presque plus maintenant. Mais, j'ai envie de dire que pendant deux ans, j'ai beaucoup, beaucoup discuté avec tout le monde et l'équipe de nuit, beaucoup. Et puis, je m'intègre et puis après on a essayé de construire des choses. » En tant qu'ex-syndicaliste convaincue, très sensible aux conditions de travail des personnels, elle maintient dans son rôle de cadre une posture d'ouverture au dialogue avec l'équipe de soins. Son style de management très humaniste s'accompagne d'un fort sentiment de responsabilité tant vis-à-vis des patients que des salariées. Cette approche la conduit rapidement à prendre en charge une grande part du fonctionnement de l'établissement, d'autant que Mr Legrand ne cherche guère à se mettre en avant tant à l'intérieur qu'à l'extérieur de l'hôpital, lui déléguant volontiers de nombreuses tâches.

Quinze ans après son arrivée, Mme Joly est aujourd'hui une actrice centrale, polyvalente, mais, en tant qu'unique cadre infirmier, quelque peu isolée dans l'organisation. Elle assure à elle seule la constitution mensuelle des plannings de tous les personnels soignants et gère le « pool» de contractuelles remplaçantes que le directeur parvient à financer. Elle est sollicitée très fréquemment pour régler des conflits entre salariées. Il lui faut aussi «faire du social», c'est-à-dire venir en aide moralement, mais aussi parfois concrètement aux agents confrontés à de plus ou moins graves difficultés familiales, de santé, psychologiques... Il lui revient également de gérer les entrées et les sorties des patients et résidents (en cas de retour à domicile ou de décès) et, par là même, de prendre en charge les relations avec les familles et 
de répondre à leurs questions. Mme Joly participe également à tous les groupes de travail et instances officielles et se trouve très souvent en charge de la rédaction des dossiers, rapports ou demandes de financement. Elle assure également l'interface entre les médecins et l'établissement, en cas de problème ou pour les assister dans leurs tâches administratives (dossiers des patients, codification des actes médicaux...). Elle est par ailleurs en contact avec de nombreux partenaires extérieurs et représente l'établissement dans le cadre de réunions, journées d'études, colloques... Elle est notamment intervenue, fin 2006, dans le cadre d'une manifestation publique organisée par l'ARH sur le thème de la gestion des ressources humaines qui a rassemblé de nombreux cadres soignants et administratifs des établissements de la région. Le CHU de $\mathrm{R}$. avait présenté à cette occasion la mise en place du Collège cadres. Mme Joly, qui connaît bien la situation des cadres du CHU pour y avoir travaillé, commente en ces termes cette intervention : «J'ai encore plein de copines qui sont cadres au CHU, c'est de la merde hein, c'est de l'esbroufe, ça sert à rien, tout ça c'est des conneries ! C'est pipeau, c'est de l'esbroufe, c'est de la paillette, c'est de la merde tout ça! C'est de la merde ! Au CHU, ils sont tous en train de souffrir, les cadres, ils ont qu'un truc à faire, c'est fermer leur gueule ! C'est... ils sont pressurisés, ils sont... C'est de la manipulation, c'est de la poudre aux yeux, c'est rien ! Et puis, il y a plein de choses qui ne se sont pas dites !»

Ces propos ont au moins le mérite de la clarté. Ils apportent un contrepoint aux discours des responsables du CHU sur le Collège cadres et tendent à suggérer que ce dispositif relèverait d'une stratégie de la direction visant à inculquer aux cadres un sentiment d'appartenance à un groupe distinct des autres salariés, appelé à diffuser la logique managériale auprès des personnels. La participation à une entité collective spécifique pourrait notamment contribuer à mobiliser subjectivement les cadres de proximité, qui expriment parfois un sentiment de malaise, se sentant pris en tenaille entre des exigences idéales et donc irréalistes de la direction et des difficultés de fonctionnement et de moyens sur le terrain qui les plongent dans des contradictions insolubles. (Dujarier, 2006).

Mme Joly n'est pas confrontée à de telles exigences. Sa charge de travail est certes très lourde, mais elle dispose d'une grande autonomie, au moins dans la gestion des affaires courantes des services. Elle ne peut effectivement pas échapper aux contraintes gestionnaires de l'ARH, mais elle évolue dans un univers de travail où les relations interpersonnelles demeurent relativement conviviales, tant avec le personnel soignant et administratif, qu'avec le directeur. La force du collectif de travail fondé sur un réseau d'interconnaissance d'une grande densité, l'éloignement géographique par rapport à l'ARH, un établissement dit à taille humaine avec un petit effectif de salariés et un style de management de la direction appartenant à l' « ancienne génération », tous ces facteurs font sans conteste de l'Hôpital local du Littoral un îlot de résistance face à la généralisation de la rationalisation des organisations par le biais des nouvelles logiques gestionnaires. Une inquiétude règne cependant dans les services. Mme Joly a en effet décidé de faire valoir ses droits à la retraite en juillet 2008. «On va perdre gros!» s'exclame plus d'un agent, pressentant sans doute qu'ils peuvent être rattrapés par un monde qu'ils parviennent encore à tenir à distance.

\section{Conclusion}

Sous leur forme présente de «vrais » cadres, différents des encadrants de soin de la tradition hospitalière, les cadres de santé sont une catégorie relativement nouvelle, mal assurée de son identité et peu dotée de référents collectifs spécifiques. D'une certaine façon, le secteur de la santé semble reproduire à trente ou cinquante ans d'intervalle les hésitations et les difficultés 
qui ont marqué la construction de la catégorie des cadres dans la société française : soutenue par le pouvoir de l'Etat et du patronat, elle peinait néanmoins à fédérer des professionnels très disparates et à se doter d'institutions propres (Boltanski, 1982). Du reste, il n'était pas rare que les entreprises se dotent de collèges de cadres dans les années 1960 (Benoît et Maurice, 1960), moyen à la fois d'aider les cadres à trouver leur place entre le monde de la direction et celui des exécutants, et de donner vie à des instances collectives intégrées à l'entreprise tout en restant extérieures aux syndicats.

L'hypothèse que l'émergence historique du personnage social du cadre, au début du XXe siècle, correspond à une phase spécifique du mouvement de rationalisation, semble ainsi confirmée par l'extension de la catégorie à des univers, comme celui de la santé, auxquels elle était restée extérieure, et dans lesquels elle fait surgir, à la faveur d'une vigoureuse mise en conformité avec les principes de la rationalité managériale, des personnages nouveaux, incertains, fragilement insérés dans leur être collectif, et dans certains cas attachés à des logiques d'action antinomiques avec cette même rationalité managériale. Un parallèle intéressant pourrait être fait avec les cadres d'entreprises publiques confrontés à la privatisation et à la pression du marché : les nouvelles règles du jeu les portent en avant, faisant d'eux la cheville ouvrière du changement, mais c'est parfois à leur corps défendant ou, comme à l'Hôpital local, en s'efforçant de s'en préserver - qu'ils le conduisent.

\section{Bibliographie}

Acker Françoise, 2003, «Les infirmières: une profession en crise ?» in La crise des professions de santé, Jean de Kervasdoué (dir.), Paris, Dunod.

Beaud Stéphane, Weber Florence, 1997, Guide de l'enquête de terrain, Paris, La Découverte.

Benamouzig Daniel, 2006, La santé au miroir de l'économie. Paris, PUF.

Benoit Odile, Maurice Marc (1960), «Groupes professionnels et relations collectives de travail dans une entreprise », Sociologie du travail, ${ }^{\circ} 2$, p. 151-169.

Chéronnet Hélène. 2006) Statut de cadre et culture de métier, La structuration des fonctions d'encadrement dans le secteur de l'éducation spécialisée, L'Harmattan, Coll. Le travail du social

Dujarier Marie-Anne, 2006, L’idéal au travail, Paris, PUF.

Féroni Isabelle, Kober-Smith Anémone, 2005, «La professionnalisation des cadres infirmiers : l'effet de l'action publique en France et en Grande-Bretagne », Revue française de sociologie, 46-3.

Foucault Michel, 1961, Histoire de la folie à l'âge classique, Paris, Gallimard.

Gadea Charles, 2003, Les cadres en France. Une énigme sociologique, Paris, Belin. 
Jounin Nicolas, Wolf Loup, 2006, Entre fonctions et statuts,les relations hiérarchiques dans les établissements de santé. DREES, Série Etudes, Documents de travail, $\mathrm{n}^{\circ} 64$ - octobre

Le Lan Romuald, 2006, Enquête sur les conditions et l'organisation du travail auprès d'actifs des établissements de santé. DREES, Série Statistique, Documents de travail n 102.

Metzger Jean-Luc ; Scwheyer François-Xavier (2005). Entre profession, organisation et marché : le cas des ingénieurs biomédicaux hospitaliers. Revue française des affaires sociales, $\mathrm{n}^{\circ} 1$, janvier-mars 2005, pp. 183-205.

Moisdon Jean-Claude, Tonneau Dominique, 1999, La démarche gestionnaire à l'hôpital, 1Recherches sur la gestion interne, Paris, Editions Seli Arslan SA.

Picon Antoine. 1998 L'invention de l'ingénieur moderne. L'Ecole des Ponts et chaussées 1747-1851. Paris, Presses de l'Ecole nationale des Ponts et Chaussées.

Renahy Nicolas, 2005, Les gars du coin. Enquête sur une jeunesse rurale, Paris, La Découverte.

Retière Jean-Noël, 2003, Autour de l'autochtonie. Réflexions sur la notion de capital social populaire, Politix, vol. 16, $\mathrm{n}^{\circ} 63$.

Serré Marina, 2002, «De l'économie médicale à l'économie de la santé. Genèse d'une discipline scientifique et transformations de l'action publique», Actes de la recherche en sciences sociales, $\mathrm{n}^{\circ} 143$, pp. 68-79.

Sainsaulieu Ivan, 2003, Le malaise des soignants, Paris, l'Harmattan

Vega Anne, 2004, Une ethnologue à l'hôpital. L'ambiguïté du quotidien infirmier, Editions des archives contemporaines. 